\title{
Electron Diffraction Analysis of 2D Protein Crystals with Large $d_{\mathrm{hkl}}$ Spacing
}

\author{
Peng Li*, Marek Malac ${ }^{*}, \diamond$ John Paul Glaves*, Howard Young ${ }^{*}$, \\ * National Institute for Nanotechnology, Edmonton, 11421 Saskatchewan Drive, T6G 2M9, Canada. \\ ${ }^{\diamond}$ Department of Physics, University of Alberta, Edmonton, T6G 2G7, Canada. \\ - Department of Biochemistry, University of Alberta, Edmonton, Alberta, T6G 2H7 Canada.
}

Electron diffraction (ED) allows reliable identification of structure and orientation of crystalline samples. For biological/protein crystals with lattice $\left(d_{\mathrm{hkl}}\right)$ from a few $\mathrm{nm}$ to a few tens $\mathrm{nm}$ the microscope camera length needs to be significantly increased. Thus, Fourier Transform (FT) of HRTEM images rather than ED have generally been used to retrieve structural information from such samples. Advantages of ED over HRTEM imaging include: 1) reduced irradiation dose; 2) ED can tolerate increased sample thickness; 3 ) the intensity of the ED spots is not affected by lens aberrations; 4) ED can tolerate sample drift [1]. Typical TEM lens settings are for camera length $L=10$ to $300 \mathrm{~cm}$, a good choice for a $d_{\mathrm{hkl}}$ from $0.1 \mathrm{~nm}$ to $1 \mathrm{~nm}$ acquired at CCD camera or at the film plane. Here we show electron diffraction patterns from biological 2D crystals with large $d_{\mathrm{hkl}}$ (lattice parameter $a=34$ $\mathrm{nm}, b=7.1 \mathrm{~nm}$ ) in a JEOL 2200 FS TEM. Furthermore we show tomography results that demonstrate that the $2 \mathrm{D}$ crystals exhibit significant bending.

The 2D protein crystals are prepared from proteoliposome vesicles of co-reconstituted sarcoplasmic reticulum $\mathrm{Ca} 2+-\mathrm{ATPase}$ and phospholamban proteins [2]. The 2D crystals lateral dimensions exceed $500 \mathrm{~nm}$. In addition to the large $d_{\mathrm{hkl}}$ of the lattice of the 2D protein crystals, inherent disorder and radiation sensitivity limit information transfer based on HRTEM alone. Standard imaging, FT analysis, and lattice unbending of crystals is limited by poor phase residuals and low amplitudes for spacing below about $0.8 \mathrm{~nm}$.

The maximum standard camera length of the JEOL $2200 \mathrm{FS}$ is $250 \mathrm{~cm}$, too small to obtain ED from the $2 \mathrm{D}$ crystals. In free lens control mode, longer camera lengths can be achieved by increasing excitation of intermediate lens I 2 and I4 and decreasing I3 excitation while using I1 to keep the diffraction pattern in focus. Isochromaticity can be maintained to obtain energy-filtered ED.

Figure 1 shows an energy-filtered HRTEM image of a negatively-stained crystal, with its FT and a low magnification bright field image shown in inserts. Figure 2 shows an ED of a crystal recorded on a CCD camera with approximately $\mathrm{L}=1300 \mathrm{~cm}$. Longer camera lengths can be achieved on an image plate media located at the bottom of the microscope without resorting to Migley's technique [3].

Tomography of a negatively-stained $2 \mathrm{D}$ crystal was also performed to visualize crystal bending. The sample was tilted from -60 to 60 degrees with 2 degree step. Even though the crystal was negatively stained, the sample was kept at cryo temperature $\left(-180 \mathrm{C}\right.$ with $\left.\mathrm{LN}_{2}\right)$ to minimize beam damage. The crystal lattice was still well resolved following collection of the tilt series. Reconstruction was done by JEOL TEMography ${ }^{\mathrm{TM}}$ [4]. Figure 3 shows an image of the crystal at 0 degree tilt and Figure 4 shows two views of the reconstructed tomogram. Figure 4 clearly shows that the crystal is bent, which provides a framework for validating traditional methods structure determination by HRTEM imaging. Some of our future goals include: zero-loss (energy-filtered) electron diffraction, and tilted diffraction series of 2D protein crystals in the frozen-hydrated state.

References:

[1] M. Malac, M. Beleggia, Y. Taniguchi, R. Egerton, Y. Zhu, Ultramicroscopy 109, p.14 (2009).

[2] D.L. Stokes, A.J. Pomfret, W.J. Rice, J.P Glaves, HS Young, Biophysical Journal (90), p. 4213-4223 (2006).

[3] P. A. Migley, Ultramicroscopy 76, p. 91 (1999).

[4] www.temography.com 


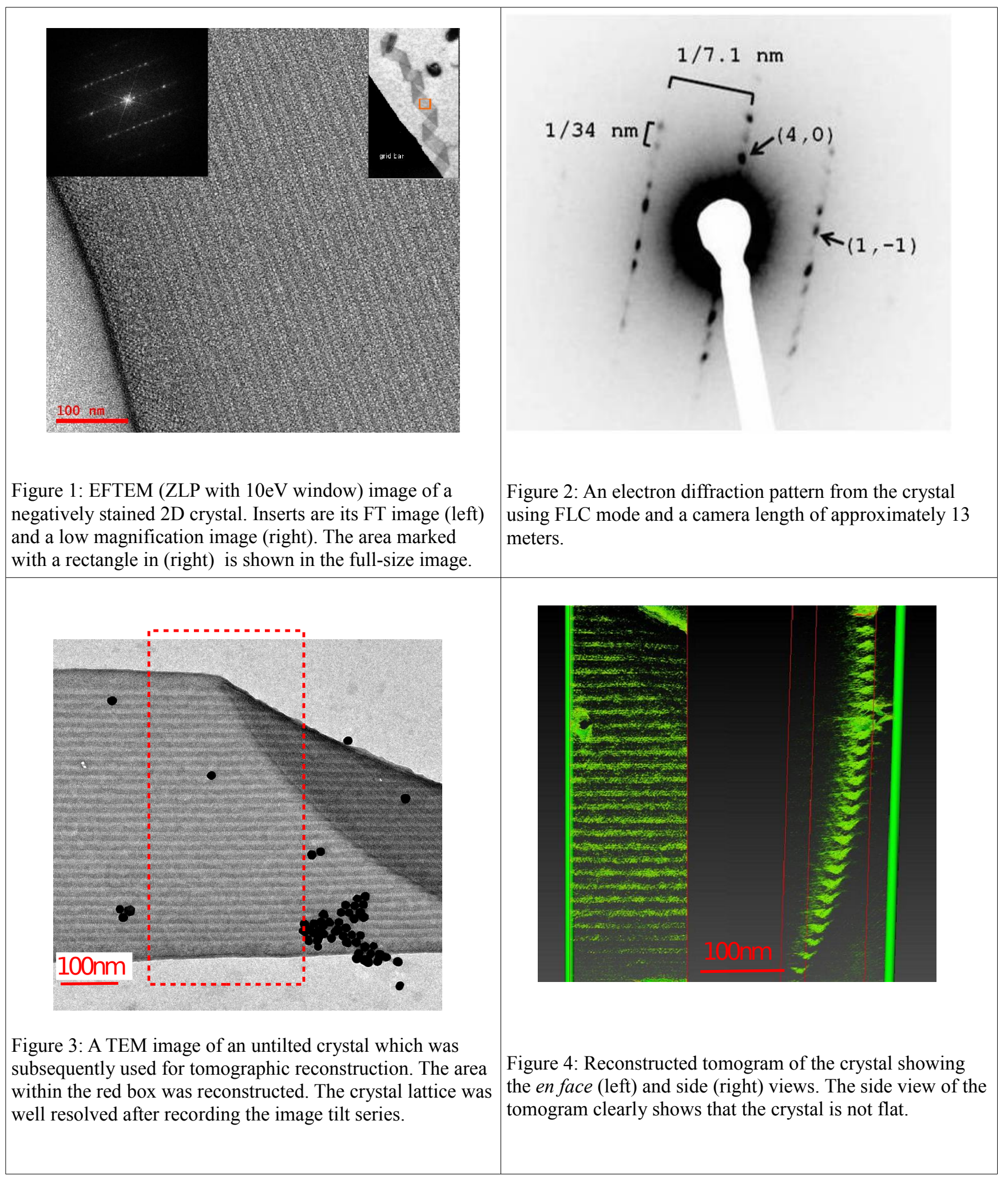

